\title{
Non-hermitian susy hydrogen-like Hamiltonians with real spectra
}

\author{
Oscar Rosas-Ortiz ${ }^{1}$ and Rodrigo Muñoz ${ }^{2}$ \\ Departamento de Física, CINVESTAV-IPN, A.P. 14-740, 07000 México D.F., Mexico
}

\begin{abstract}
It is shown that the radial part of the hydrogen Hamiltonian factorizes as the product of two not mutually adjoint first order differential operators plus a complex constant $\epsilon$. The 1-susy approach is used to construct non-hermitian operators with hydrogen spectra. Other non-hermitian Hamiltonians are shown to admit an extra 'complex energy' at $\epsilon$. New self-adjoint hydrogen-like Hamiltonians are also derived by using a 2-susy transformation with complex conjugate pairs $\epsilon, \bar{\epsilon}$.
\end{abstract}

PACS: 03.65.Ge; 03.65.Fd; 03.65.Ca

Keywords: Darboux transformation, supersymmetric quantum mechanics, complex potentials

\section{Introduction}

The supersymmetric Quantum Mechanics (SUSY QM) has grown on the factorization and intertwining methods [1] applied to transform the physical Hamiltonians. It yields new exactly solvable potentials which are either strictly isospectral to the initial one because of broken SUSY, or almost isospectral due to unbroken SUSY (see the recent reviews [2].) The higher order SUSY QM amended the conviction that the excited states can not be used to generate non-singular susy partners [3, 4, 5, 6]. Some applications deal with singular [7, soliton-type [8, periodic [9] and other potentials [10]. Of special interest is the confluent algorithm [8,11] for which the second order procedure is applied to add a single level at an arbitrary point of the energy axis. However, almost all the works on the subject make use of transformation functions with the real factorization constants $\epsilon$ and with the factor-operators being always mutually adjoint.

The case of complex $\epsilon$ has not been studied to the desired extent. Exceptional cases seem 12, 13, 14, where the 2-susy treatment with $\epsilon \in \mathbb{C}$ is formulated to obtain either hermitian or non-hermitian susy partners of a given initial Hamiltonian. Indeed, one of our purposes is to show that the Hamiltonians $H$ can be factorized as the product of two not mutually adjoint first order operators $A, B$, plus a complex constant even for self-adjoint $H$. The method is not limited to the Hamiltonians

\footnotetext{
${ }^{1}$ Corresponding Author. E-mail: orosas@fis.cinvestav.mx

${ }^{2} \mathrm{E}$-mail: rodrigom@fis.cinvestav. $\mathrm{mx}$
} 
possessing the lower spectral bound; it can also yield non-hermitian Hamiltonians with complex potentials. Although the typical Hamiltonians of QM are hermitian, non-hermitian ones appear in Molecular Physics and Quantum Chemistry [15], Superconductivity [16], Quantum Field Theory [17] and other domains [18. The fact that they admit real eigenvalues for which the associated eigenfunctions are square-integrable [19] has been the basis of recent studies on PT-symmetry [20,21], pseudo-hermiticity [22,23] and diverse physical models 24].

In this paper we illustrate these facts by constructing the hermitian and nonhermitian susy partners of the radial part $\left(H_{\ell}\right)$ of the hydrogen Hamiltonian. Even though our non-hermitian operators are not $P T$-invariant, we shall see that a class of them has real eigenvalues identical to the hydrogen energies. The reality of the spectrum in this case is due to the breaking of supersymmetry.

It will be shown that there is another class of non-hermitian operators having an extra square-integrable eigenfunction associated with $\epsilon \in \mathbb{C}$. In this case, the 'complex energy' $\epsilon$ arises from the unbroken supersymmetry and, up to now, does not have a well established physical meaning (but see [25]). Unlike the phase-equivalent complex potentials [14, the new 'bound state' associated with $\epsilon$ is nodeless. Moreover, in counterdistinction with the formalism of PT-symmetry and pseudo-hermiticity, where complex energies appear in conjugate pairs, it turns out that $\bar{\epsilon}$ does not belong to the spectrum of the susy partner of $H_{\ell}$ generated through $\epsilon$.

In general, we shall see that the susy transformation is adequate to analytically determine normalizable eigenfunctions of non-hermitian Hamiltonians, including those with the complex energies. In this sense, our 'complex susy transformation' seems an analytical complement of the numerical techniques previously reported (see [14] and references therein.) The eigenfunction connected with $\epsilon$ is then removed by iterating the procedure in order to construct hermitian 2-susy partners of $H_{\ell}$.

The paper is organized as follows: Section 2 introduces the atypical factorizations $H_{\ell}=A B+\epsilon$, where $\epsilon \in \mathbb{C}$ and the first order differential operators $A$ and $B$ are not mutually adjoint. Sections 3 and 4 are devoted to the construction of nonhermitian 1-susy and hermitian 2-susy partners, $\mathrm{H}(\zeta)$ and $\widetilde{H}$ respectively, of $H_{\ell}$. Final remarks and discussion are given in Section 5.

\section{The complex-type factorization method}

Let us consider a single electron in the field produced by a nucleus with $Z$ protons. We shall use $\mathcal{E}=Z / 2 r_{B}$ and $r_{B}=\hbar^{2} / Z e^{2} m$ for the units of energy and coordinates respectively. The corresponding time-independent Schrödinger equation reduces to $H_{\ell} \psi_{n, \ell}(r)=E_{n} \psi_{n, \ell}(r)$, with solutions

$$
\psi_{n, \ell}(r)=C_{n, \ell} r^{\ell+1} e^{-r / n} F_{1}(\ell+1-n, 2 \ell+2 ; 2 r / n), \quad E_{n}=-1 / n^{2}
$$


where $\mathbb{N} \ni n=\ell+s+1 ; \ell=0,1,2, \ldots, n-1 ; s \in \mathbb{Z}^{+} ; C_{n, \ell}$ is the normalization constant, ${ }_{1} F_{1}(a, c, z)$ is the Kummer's function and $L^{2}\left(\mathbb{R}^{+}, 4 \pi\right) \ni \psi_{n, \ell}(r) \equiv r R_{n, \ell}(r)$, with an inner product defined by $\langle\psi, \phi\rangle=4 \pi \int_{0}^{+\infty} \bar{\psi}(r) \phi(r) d r<\infty$ and boundary conditions at $r=0: \psi(0)=0, \psi^{\prime}(r)=R(0)$. The effective potential $V_{\ell}(r)$ has the domain $\mathcal{D}_{V}=[0, \infty)$ and

$$
H_{\ell} \equiv-\frac{d^{2}}{d r^{2}}+V_{\ell}(r)=-\frac{d^{2}}{d r^{2}}+\frac{\ell(\ell+1)}{r^{2}}-\frac{2}{r} .
$$

The Hamiltonian (2) is factorized as follows:

$$
H_{\ell}=A B+\epsilon
$$

where the factorization constant is a complex number $\mathbb{C} \ni \epsilon:=\epsilon_{1}+i \epsilon_{2} ; \epsilon_{1}, \epsilon_{2} \neq 0 \in \mathbb{R}$ and the first order operators $A, B$, are not mutually adjoint (compare [6, 26]):

$$
A:=-\frac{d}{d r}+\beta(r), \quad B:=\frac{d}{d r}+\beta(r)
$$

with $\beta$ a complex-valued function fulfilling

$$
-\beta^{\prime}(r)+\beta^{2}(r)+\epsilon=V_{\ell}(r) .
$$

The Riccati equation (15) is solved by means of the logarithmic transformation $\beta(r)=$ $-\frac{d}{d r} \ln u(r)$ for which $u$ is the most general eigenfunction of $H_{\ell}$ (not necessarily normalizable) belonging to $\epsilon \equiv-k^{2} ; \mathbb{C} \ni k=k_{1}+i k_{2} ; k_{1}, k_{2} \in \mathbb{R}$ :

$$
\begin{aligned}
& u(r)=r^{\ell+1} e^{-k r} f(r) ; \\
& f(r):=\alpha_{1} F_{1}(\ell+1-1 / k, 2 \ell+2,2 k r)+\zeta U(\ell+1-1 / k, 2 \ell+2,2 k r)
\end{aligned}
$$

where $\alpha$ and $\zeta$ are complex constants and $U(a, c, z)$ is the logarithmic hypergeometric function. The global behaviour of these $u$-functions is analized in Appendix.

Hence, for the $\beta$-function we have:

$$
\beta(r)=-\frac{\ell+1}{r}+k+\Omega(r) ; \quad \Omega(r):=-\frac{d}{d r} \ln f(r) .
$$

A convenient expression for $\beta_{1}(r), \beta_{2}(r)$, the real and imaginary parts of $\beta(r)$ respectively, can be found in Appendix.

\section{New complex hydrogen-like potentials}

Let us consider the value $\epsilon$ fixed. By convenience we shall make explicit the dependence of $\Omega$ on $\zeta$. Now, let us reverse the order of the factors in (3):

$$
B A+\epsilon=-\frac{d^{2}}{d r^{2}}+V_{\ell+1}(r)+2 \Omega^{\prime}(r ; \zeta) \equiv-\frac{d^{2}}{d r^{2}}+\mathrm{V}(r ; \zeta):=\mathrm{H}(\zeta)
$$


where $\mathrm{H}(\zeta)$ is a non-hermitian second order differential operator and we have used (5) and (7). The next step is to solve the related eigenvalue equation:

$$
\mathrm{H}(\zeta) \Psi=\lambda \Psi, \quad \lambda=\lambda_{1}+i \lambda_{2}, \quad \lambda_{1}, \lambda_{2} \in \mathbb{R}
$$

The dependence of $\Psi$ and $\lambda$ on $\zeta$ will be dropped for simplicity. Notice that equations (3) and (8) imply an intertwining between the Hamiltonian $H_{\ell}$ and the nonhermitian operator $\mathrm{H}(\zeta)$ :

$$
\mathrm{H}(\zeta) B=B H_{\ell}, \quad H_{\ell} A=A \mathrm{H}(\zeta)
$$

Thereby, one sees that $\Psi \propto B \varphi$ is a solution of (9) if $\varphi$ satisfies $H_{\ell} \varphi=\lambda \varphi$, while $A$ reverses the action of $B$. Now, the general form of $\varphi$ is obtained by taking $\epsilon=-k^{2}$ for $\lambda=-\kappa^{2}$, and $u(r)$ for $\varphi(r)$ in (6). Hence, we have:

$$
\varphi(r)=r^{\ell+1} e^{-\kappa r}\left\{C_{1} F_{1}(\ell+1-1 / \kappa, 2 \ell+2,2 \kappa r)+D U(\ell+1-1 / \kappa, 2 \ell+2,2 \kappa r)\right\}
$$

with $C$ and $D$ arbitrary complex constants. Therefore

$$
\Psi \propto B \varphi=\frac{W(u, \varphi)}{u}
$$

We are looking now for the constraints on $\alpha, \zeta, \lambda, C$ and $D$ leading to squareintegrable $\Psi$. First, consider $\lambda \neq \epsilon($ i.e., $\kappa \neq k)$; the behaviour of $\Psi$ near the origin is

$$
\Psi(r \sim 0) \propto \begin{cases}-D \frac{(2 \ell+1)}{(2 \kappa)^{2 \ell+1}} \frac{\Gamma(2 \ell+1)}{\Gamma(\ell+1-1 / \kappa)} \frac{1}{r^{\ell+1}}, & \zeta=0, \alpha \neq 0 \\ D \frac{(k-\kappa)}{(2 \kappa)^{2 \ell+1}} \frac{\Gamma(2 \ell+1)}{\Gamma(\ell+1-1 / \kappa)} \frac{1}{r^{\ell}} & \zeta \neq 0, \text { arbitrary } \alpha\end{cases}
$$

Thus, $\Psi$ becomes divergent at $r=0$ except if either (I) $\lambda=-\kappa^{2}$ is real and $\kappa^{-1}=\ell+s+1, s \in \mathbb{Z}^{+}$, or (II) $\lambda$ is complex but $D=0$. Let us pay some attention to these conditions

CASE I (real $\lambda$ ): Let us fix $\mathbb{R} \ni \kappa^{-1}=\ell+s+1=n, n \in \mathbb{N}$. In this case, in (11) ${ }_{1} F_{1}(a, c ; z)$ and $U(a, c ; z)$ are essentially the same function. Therefore, one can take $D=0$ and $C=C_{n, \ell}$ (see equation (1)), so $\varphi(r)=\psi_{n, \ell}(r)$ and the functions (12) behave asymptotically as $\Psi(r) \propto \psi_{n, \ell}(r)_{r \rightarrow \infty}$. On the other hand, a straightforward calculation shows that these functions obey the following boundary conditions at $r=0: \Psi(0, \zeta)=R(0) ; \Psi^{\prime}(0, \zeta=0)=-\delta_{\ell 0} ; \Psi^{\prime}(0, \zeta \neq 0)=-\ell\left(\frac{\delta_{\ell 0}}{r}+\delta_{\ell 1}\right)$, with $\delta_{\ell n}$ the kronecker delta. Thus

$$
\Psi(r ; \zeta) \propto\left[k-\frac{1}{n}+\frac{d}{d r} \ln \left(\frac{{ }_{1} F_{1}(\ell+1-n, 2 \ell+2 ; 2 r / n)}{f(r)}\right)\right] \psi_{n, \ell}(r)
$$

are square-integrable eigenfunctions of $\mathrm{H}(\zeta)$ with the real eigenvalues $\lambda=-1 / n^{2}=$ $E_{n}$. 
CASE II (complex $\lambda)$ : For $\kappa \in \mathbb{C}$ and $D=0$ the function (12) behaves asymptotically as follows:

$$
\Psi(r) \sim \begin{cases}C \frac{(\kappa \mp k)}{(2 \kappa)^{\ell+1+1 / \kappa}} \frac{\Gamma(2 \ell+2)}{\Gamma(\ell+1-1 / \kappa)} \frac{e^{\kappa r}}{r^{1 / \kappa}} & \kappa_{1}>0,\left\{\begin{array}{l}
k_{1}>0 \\
k_{1}<0
\end{array}\right. \\
\mp C \frac{(k \pm \kappa)}{(-2 \kappa)^{\ell+1-1 / \kappa}} \frac{\Gamma(2 \ell+2)}{\Gamma(\ell+1+1 / \kappa)} \frac{e^{-\kappa r}}{r^{-1 / \kappa}} & \kappa_{1}<0,\left\{\begin{array}{l}
k_{1}>0 \\
k_{1}<0
\end{array}\right.\end{cases}
$$

which always diverges for $r \rightarrow \infty$. Hence, there is no function $\Psi \in L^{2}\left(\mathbb{R}^{+}, 4 \pi\right)$ solving (9) for a complex $\lambda \neq \epsilon($ i.e. $\kappa \neq k$ ) and $D=0$.

The formula (14) therefore gives all square integrable solutions $\{\Psi(r ; \zeta)\}$ of (9) for $\lambda \neq \epsilon$. Concerning the case $\lambda=\epsilon$, we see from equation (8) that any $\Psi_{\epsilon}(r)$ in the one-dimensional kernel of $A$ is an eigenfunction of $\mathrm{H}(\zeta)$ belonging to $\epsilon$. After a simple calculation one gets

$$
\Psi_{\epsilon}(r) \propto \frac{1}{u(r)} .
$$

This function can be in $L^{2}\left(\mathbb{R}^{+}, 4 \pi\right)$ for appropriate values of $\alpha$ and $\zeta$. In such a case, $\Psi_{\epsilon}$ is an extra square-integrable eigenfunction of $\mathrm{H}(\zeta)$ associated with $\epsilon \epsilon$ $\mathbb{C}$. Finally, a straighforward calculation shows that, although $\left\{\Psi(r ; \zeta), \Psi_{\epsilon}(r)\right\}$ are elements of $L^{2}\left(\mathbb{R}^{+}, 4 \pi\right)$, they do not form an orthogonal set. A discussion on this kind of properties of the inner product is given in [24]. The next subsections analize these conditions and classify the resulting potentials according to their spectra.

\subsection{The real spectrum}

From Figure 1a, one sees that the behaviour of the complex function $\mathrm{V}(r ; \zeta)$, for $\alpha \neq 0$ and $\zeta=0$, is given by

$$
\mathrm{V}(r ; \zeta=0) \sim \begin{cases}V_{\ell+1}(r), & \text { for } r \sim 0, \\ 0, & r \rightarrow \infty .\end{cases}
$$

Consistently with the case $(\mathbf{A})$ of Appendix, the function $\Psi_{\epsilon}(r)$ diverges at $r=0$. Hence, $\Psi_{\epsilon}(r)$ is not in $L^{2}\left(\mathbb{R}^{+}, 4 \pi\right)$ and the discrete spectrum $\sigma_{d}\left(\mathrm{H}_{\ell+1}\right)$ of $\mathrm{H}(\zeta=0)$ is exactly the same as that of the hydrogen atom $\sigma_{d}\left(\mathrm{H}_{\ell+1}\right)=\sigma_{d}\left(H_{\ell}\right)$. Figure $1 \mathrm{~b}$ depicts the behaviour of $\psi_{n, \ell}$ and the related function (14) for one of the excited states.

Another class of complex potentials $\mathrm{V}(r ; \zeta)$ sharing the same spectrum as $H_{\ell}$ is obtained by considering the case $(\mathbf{B})$ of Appendix. 


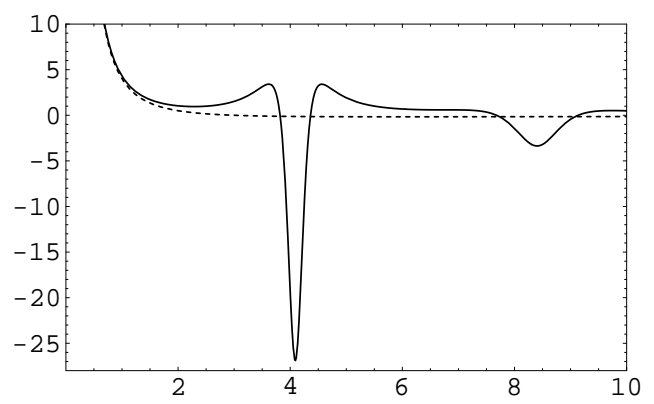

(a)

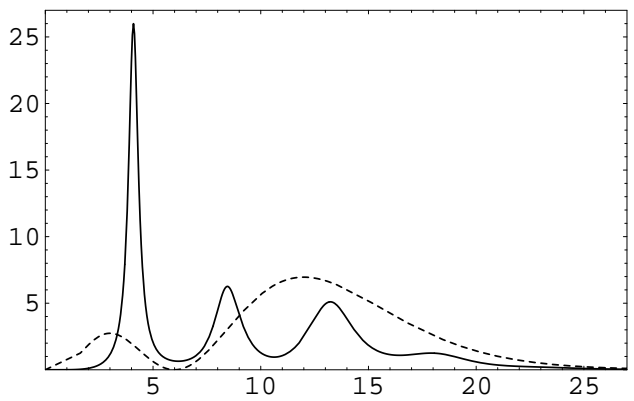

(b)

Figure 1: (a) The potential $V_{\ell+1}(r)$ (dashed curve) and the real part of $\mathrm{V}(r ; \zeta)$ for $\ell=1$, $\epsilon=-(0.1+i 0.5)^{2}, \alpha=1$, and $\zeta=0$. (b) The corresponding unnormalized $\left|\psi_{3,1}(r)\right|^{2}$ (dashed curve) and its susy partner $|\Psi(r ; \zeta=0)|^{2}$ for $E_{3}=-1 / 9$ and the same values of the parameters.

\subsection{Complex potentials admitting complex 'energies'}

Let us consider $\zeta \neq 0$. Then the complex function $\mathrm{V}(r ; \zeta)$ behaves as shown on Figure 2a, i.e.:

$$
\mathrm{V}(r ; \zeta \neq 0) \sim \begin{cases}V_{\ell-1}(r), & \text { for } r \sim 0, \text { arbitrary } \alpha \\ 0, & r \rightarrow \infty .\end{cases}
$$

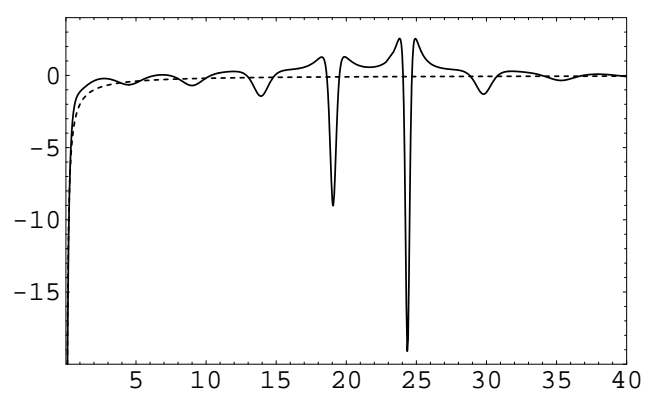

(a)

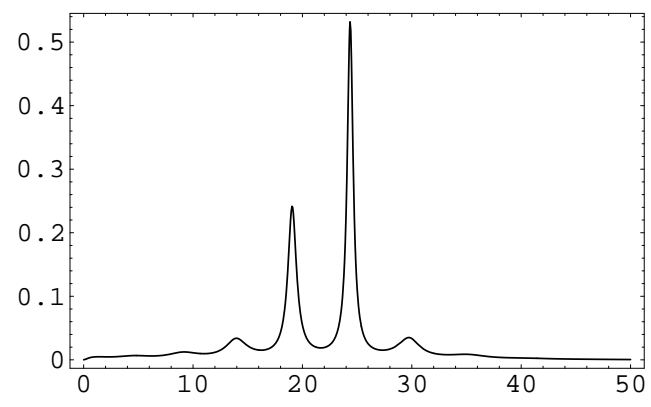

(b)

Figure 2: (a) The potential $V_{\ell-1}(r)$ (dashed curve) and the real part of $\mathrm{V}(r ; \zeta)$, for $\ell=1$, $\epsilon=-(0.1+i 0.5)^{2}, \alpha=1$, and $\zeta=0.5+i 0.5$. (b) The corresponding unnormalized $\left|\Psi_{\epsilon}(r)\right|^{2}$ for the same values of the parameters

The case $(\mathbf{D})$ of Appendix shows that, for $(\alpha, \zeta) \in \mathbb{C}_{0}$ and $\theta_{\ell}(k) \neq 0$, the function $\Psi_{\epsilon}(r)$ behaves as

$$
\Psi_{\epsilon}(r) \sim \begin{cases}\frac{(2 k)^{2 \ell+1}}{\zeta} \frac{\Gamma(\ell+1-1 / k)}{\Gamma(2 \ell+1)} r^{\ell} & r \sim 0 \\ \frac{(2 k)^{\ell+1+1 / k}}{\alpha} \frac{\Gamma(\ell+1-1 / k)}{\Gamma(2 \ell+2)} r^{1 / k} e^{-k r} & r \rightarrow \infty, k_{1}>0 \\ \frac{(2 k)^{\ell+1-1 / k}}{\theta_{\ell(k)}} r^{-1 / k} e^{k r} & r \rightarrow \infty, k_{1}<0\end{cases}
$$

which clearly belongs to $L^{2}\left(\mathbb{R}^{+}, 4 \pi\right)$. Figure $2 \mathrm{~b}$ depicts the global behaviour of $\left|\Psi_{\epsilon}\right|^{2}$. Therefore, the discrete spectrum $\sigma_{d}\left(\mathrm{H}_{\ell-1}\right)$ of $\mathrm{H}(\zeta \neq 0)$ is given by $\sigma_{d}\left(\mathrm{H}_{\ell-1}\right)=$ $\sigma_{d}\left(H_{\ell}\right) \cup\{\epsilon\}$, provided that $(\alpha, \zeta) \in \mathbb{C}_{0}, \theta_{\ell}(k) \neq 0$. 
Finally, the case $(\mathbf{C})$ of Appendix, gives another solution in the same class.

\section{The new real hydrogen-like potentials}

We shall now extend the analysis of the previous section by intertwining $H_{\ell}$ with a new (to be determined) Hamiltonian $\widetilde{H}$ as follows

$$
\widetilde{H} \widetilde{A}=\widetilde{A} H_{\ell}
$$

where the differential operator $\widetilde{A}$ is of second order

$$
\widetilde{A}:=\frac{d^{2}}{d r^{2}}+\eta(r) \frac{d}{d r}+\gamma(r)
$$

and $\widetilde{H}$ reads

$$
\widetilde{H}:=-\frac{d^{2}}{d r^{2}}+\widetilde{V}(r)
$$

The operators (19) 201 depend implicitly on the label $\ell$. A straightforward calculation allows to express the functions $\eta$ and $\gamma$ of (19) in terms of the auto-Bäcklund transformation of the solutions of (5]) for $\epsilon_{a}$ and $\epsilon_{b}$ [6]:

$$
\begin{aligned}
& \eta(r)=-\left(\frac{\epsilon_{a}-\epsilon_{b}}{\beta_{a}(r)-\beta_{b}(r)}\right), \quad \epsilon_{a} \neq \epsilon_{b}, \\
& \gamma(r)=\beta_{b}^{\prime}(r)-\beta_{b}^{2}(r)+\eta(r) \beta_{b}(r), \quad \epsilon_{a} \neq \epsilon_{b}
\end{aligned}
$$

Thus, the second order intertwining operator $\widetilde{A}$ in (19) is expressed by two different solutions of the first order case. Moreover, it factorizes as $\widetilde{A}=a_{2} a_{1}$, where

$$
a_{1} \equiv \frac{d}{d r}+\beta_{a}=B ; \quad a_{2} \equiv \frac{d}{d r}+\eta-\beta_{a}=-A+\eta
$$

Thereby, it is easy to rewrite $\widetilde{A}$ as

$$
\widetilde{A}=(-A+\eta) B=-H_{\ell}+\epsilon+\eta B
$$

and potential (20) is obtained through

$$
\widetilde{V}(r)=V_{\ell}(r)+2 \eta^{\prime}(r)
$$

In order to get a real $\eta(r)$ in (21), 25), we consider the solution $\beta_{a}(r)$ of (15) for $\epsilon_{a} \in \mathbb{C}$ as given and, by taking $\epsilon_{b}=\bar{\epsilon}_{a}$ and $\beta_{b}(r)=\bar{\beta}_{a}(r)$ in (21), one finds

$$
\eta(r)=-\frac{\operatorname{Im}\left(\epsilon_{a}\right)}{\operatorname{Im}\left(\beta_{a}\right)} \equiv-\frac{\epsilon_{2}}{\beta_{2}(r)}=-\frac{d}{d r} \ln \omega(r)
$$


where $\omega$ is defined in (A.2) of Appendix and the labels $a$ and $b$ have been dropped from $\epsilon_{2}$ and $\beta_{2}$. Henceforth, potential (25) is real:

$$
\widetilde{V}(r)=V_{\ell}(r)-2 \frac{d^{2}}{d r^{2}} \ln \omega(r) .
$$

We are looking for potentials $\tilde{V}(r)$ defined in the same initial domain $\mathcal{D}_{V}=[0, \infty)$ (the situation when the initial domain is changed requires a different treatment, see e.g. Márquez et. al. in [7].) According to the proposition of Appendix, $\omega$ has at most one isolated zero in $\mathcal{D}_{V}$. By choosing a proper $u$, the function $\omega$ can be so constructed that its isolated zero coincides with one of the edges of $\mathcal{D}_{V}$ (compare [13]):

$$
\begin{array}{ccc}
\lim _{r \rightarrow 0} u(r)=0 & \Rightarrow & \lim _{r \rightarrow 0} \omega(r)=0 \\
\lim _{r \rightarrow \infty} u(r)=0 & \Rightarrow & \lim _{r \rightarrow \infty} \omega(r)=0
\end{array}
$$

Let us examine the consequences. First, condition (28) is satisfied if the $u$ in (6) is chosen with $\alpha \neq 0$ and $\zeta=0$, so that the potential (27) behaves as shown on Figure 3, i.e.:

$$
\widetilde{V}(r ; \alpha \neq 0 ; \zeta=0) \sim \begin{cases}V_{\ell+2}(r) & \text { for } r \sim 0, \text { arbitrary } k_{1} \\ 0 & r \rightarrow \infty, \text { arbitrary } k_{1}\end{cases}
$$

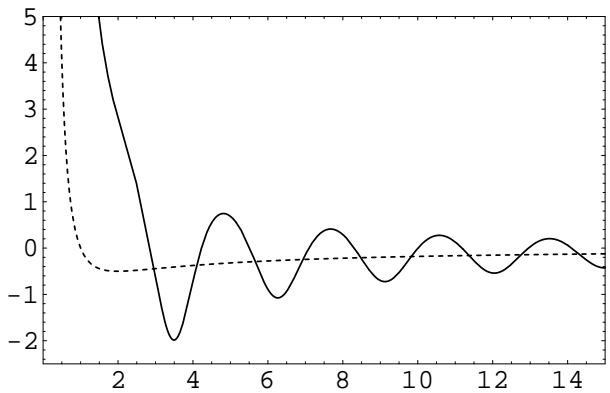

(a)

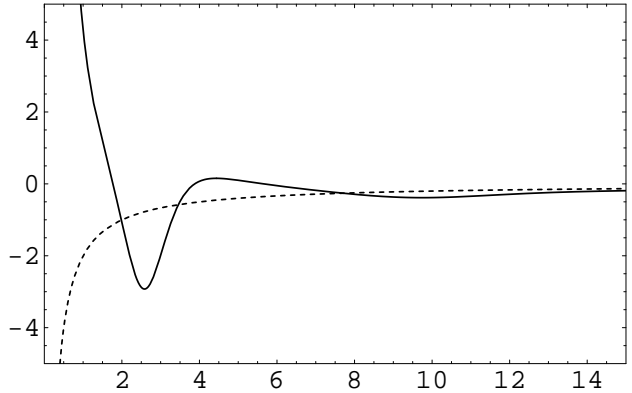

(b)

Figure 3: The initial potential $V_{\ell}(r)$ (dashed curve) and its 2-susy partner $\tilde{V}(r)$ for $\alpha=1, \zeta=0$ and (a) $\ell=1, \epsilon=-(0.01+i)^{2}$ (b) $\ell=0, \epsilon=-(0.5+i 0.1)^{2}$

On the other hand, condition (29) can be achieved for $\alpha=0, \zeta \neq 0$ and $k_{1}>0$. In this case the equation (27) leads to

$$
\widetilde{V}(r ; \alpha=0 ; \zeta \neq 0) \sim \begin{cases}V_{\ell}(r) & \text { for } r \sim 0, \\ 0 & r \rightarrow \infty .\end{cases}
$$

The real-valued potentials (30 31) resemble the hydrogen one and they could, in principle, represent physical systems as the Hamiltonians (20) are self-adjoint. The next step is to analize the new eigenvalue equation

$$
\widetilde{H} \widetilde{\psi}=\widetilde{E} \widetilde{\psi}
$$


whose solutions, by similar arguments as for the first order case, are now obtained from the linear second order transformation (see equation (18)):

$$
\widetilde{\psi}(r) \propto \widetilde{A} \psi_{n, \ell}(r)=\left(-E_{n}+\epsilon\right) \psi_{n, \ell}(r)+\eta(r, \zeta) \Psi(r, \zeta)
$$

where we have used (24) and $\Psi(r, \zeta)$ is given by (14). The corresponding boundary conditions at $r=0$ can be obtained from those of $\psi_{n, \ell}(r)$ and $\Psi(r, \zeta)$.

It is clear that the first term at the r.h.s. of (133) is in $L^{2}\left(\mathbb{R}^{+}, 4 \pi\right)$. The behaviour of the related second term is found by observing that

1) If $\alpha \neq 0$ and $\zeta=0$ then $\eta$ diverges as $r^{-1}$ at the origin while it is constant at $r=\infty$. Therefore the product $\eta(r, 0) \Psi(r, 0)$ is zero at the edges of $\mathcal{D}_{V}$ and remains finite in all $\mathcal{D}_{V}$.

2) If $\alpha=0$ and $\zeta \neq 0$ then $\eta$ is a constant at both edges of $\mathcal{D}_{V}$. Hence, $\eta(r, \zeta \neq 0) \Psi(r, \zeta \neq 0)$ is again well behaved in all $\mathcal{D}_{V}$.

Thus, the eigenfunctions $\widetilde{\psi}$ of $\widetilde{H}$ given in (33) are in $L^{2}\left(\mathbb{R}^{+}, 4 \pi\right)$ and $\widetilde{H}$ is an exactly solvable Hamiltonian with the same spectrum as the hydrogen atom.

\section{Summary and Discussion}

In this paper we have used a new type of factorization method to analyze a set of non-hermitian susy partners of the radial part of the hydrogen Hamiltonian. In order to generate the corresponding potentials we have used Darboux transformations with complex factorization constants. In contrast with PT-symmetry [19, 20, 21] and pseudo-supersymmetry [22, the breaking of supersymmetry leads to purely real spectra. However, while the pseudo-hermiticity and PT-symmetry breaking involve pairs of conjugate complex eigenvalues, the unbroken supersymmetry (for which the non-hermitian 1-susy partners are not strictly isospectral) involves just a single 'complex energy'. To be more precise, in order to add two extra eigenvalues (real or complex) to a given spectrum one applies either twice the 1-susy procedure or a single 2-susy transformation (both can be made equivalent for the case we are dealing with.) Now, if the two new energies form a complex conjugate pair and if the functions $\eta(r)$ and $\gamma(r)$ of the non-singular intertwining operator (19) are real, then the final Hamiltonian becomes self-adjoint and does not admit any complex eigenvalue, just as we have shown in Section 4.

The problem of finding normalizable solutions belonging to complex eigenvalues

for non-hermitian Schrödinger equations has been solved previously by numerical techniques in [14] and analized inside a Lie-algebraic framework in [27]. In this paper we performed an analytical study and we expect that our results complement the numerical ones. Similarly as in other non-hermitian cases discussed in the literature, the interpretation of the 'complex energies' is an open problem, though notice possible applications to the absorptive (dissipative) systems 25]. 
In general, we have shown that, the extension of the SUSY treatment to include complex factorization constants leads to results which are out of the scope of the PT-symmetry and pseudo-hermiticity. Indeed, the reality of the spectrum of the Hamiltonians $\mathrm{H}(\zeta)$ in Section 3 depends on the parameter $\zeta$, as it has been established in Subsections 3.1 and 3.2. As the non-hermitian Hamiltonians $\mathrm{H}(\zeta \neq 0)$ in Subsection 3.2 do not satisfy the theorems by Mostafazadeh [22, they are not pseudo-hermitian. On the other hand, it is not yet clear wheter the Hamiltonians $\mathrm{H}(\zeta=0)$ of Subsection 3.1 could be pseudo-hermitian or not (though a primary impression can be depicted by noticing that the non-hermiticity of $\mathrm{H}(\zeta)$ depends not on $\zeta$ but on the non-trivial imaginary part of the factorization constant $\epsilon$.) Work in this direction is in progress and will be published elsewhere.

Acknowledgements. The support of CONACyT (México), project 40888-F, is acknowledged.

\section{Appendix}

The global behavour of the eigenfunctions $u(r)$ of equation (6) can be described in terms of $\alpha, \zeta$ and the sign of $k_{1}$ :

A) If $\alpha \neq 0$ and $\zeta=0$ then $u(r)$ is zero at the origin $r=0$ while it diverges at $r=\infty$.

B) If $\alpha=0, \zeta \neq 0$ and $k_{1}>0$, then $u(r)$ diverges at the origin and tends to zero for $r \rightarrow \infty$.

C) If $\alpha=0, \zeta \neq 0$ and $k_{1}<0$, then $u(r)$ diverges at $r=0$ and $r=\infty$.

D) Let $\mathbb{C}_{0} \subset \mathbb{C} \times \mathbb{C}$ be the subset of complex pairs $\alpha \neq 0, \zeta \neq 0$, such that

$$
\zeta \neq-\alpha \frac{{ }_{1} F_{1}\left(\ell+1-1 / k, 2 \ell+2,2 k r_{0}\right)}{U\left(\ell+1-1 / k, 2 \ell+2,2 k r_{0}\right)}, \quad \forall r_{0} \in(0, \infty)
$$

If

$$
\theta_{\ell}(k):=\zeta+\alpha \frac{\Gamma(2 \ell+2)}{\Gamma(\ell+1+1 / k)} e^{ \pm i(\ell+1-1 / k) \pi}
$$

is different from zero for $(\alpha, \zeta) \in \mathbb{C}_{0}$, then $u(r)$ is free of zeros in all $\mathcal{D}_{V}$ and diverges at $r=0$ and $r=\infty$.

E) If $(\alpha, \zeta) \in \mathbb{C}_{0}, k_{1}<0$ and $\theta_{\ell}(k)=0$, then $u(r)$ diverges at the origin while $\lim _{r \rightarrow \infty} u(r)=0$ (coinciding indeed with $\left.\mathbf{B}\right)$.

The presence of zeros in these functions has been studied by means of the following

Proposition: Let $u(r) \in C^{1}\left(D_{v}\right)$ be solution of the Schrödinger equation $u^{\prime \prime}(r)=[v(r)-\epsilon] u(r)$, where $v(r)$ is a real-valued potential with domain $\mathcal{D}_{v}$ 
and $\epsilon \in \mathbb{C}$. Assume that $\mathcal{D}_{v}$ is a simply connected region of $\mathbb{R}$. If $\operatorname{Im}(\epsilon) \neq 0$, then the complex-valued function $u(r)$ admits at most one isolated zero in $\mathcal{D}_{v}$.

Proof: Let

$$
\omega(r):=\frac{W(u, \bar{u})}{2 i \operatorname{Im}(\epsilon)}
$$

where the bar denotes complex conjugation and $W(\cdot, \cdot)$ corresponds to the Wronskian of the involved functions. Clearly $\omega$ is continuous on $\mathcal{D}_{v}$ and $\omega^{\prime}(r)=$ $|u(r)|^{2} \geq 0 \forall r \in \mathcal{D}_{v}$, so $\omega(r)$ is always non-decreasing and can have either only one isolated zero or an entire interval of zero points in $\mathcal{D}_{v}$. As every zero of $u(r)$ is, necessarily, a zero of $\omega(r)$ then $u(r)$ admits at most one isolated zero there. $\diamond$

The real function $\omega(r)$ in A.2 plays a relevant role in the 2-susy approach of Section 4. A convenient expression for $\beta(r)$ in terms of $\omega$ is given by

$$
\beta(r)=\beta_{1}(r)+i \beta_{2}(r) \equiv-\frac{1}{2} \frac{d}{d r} \ln \omega^{\prime}(r)+i \epsilon_{2}\left(\frac{d}{d r} \ln \omega(r)\right)^{-1} .
$$

\section{References}

[1] E. Schrödinger, Proc. Roy. Irish Acad. A 46, 9 (1940);

R.W. Carrol 1979, Transmutation and Operator Differential Equations, NorthHolland, the Netherlands;

B.K. Bagchi 2000, Supersymmetry in Quantum and Classical Mechanics, Chapman \& Hall, New York.

[2] R. de Lima Rodriguez, e-print: hep-th/0205017 V6;

A.A. Andrianov, A.V. Sokolov, e-print hep-th/0301062

[3] A.A. Andrianov, M.V. Ioffe and V.P. Spiridonov, Phys. Lett. A 174, 273 (1993); A.A. Andrianov, M.V. Ioffe, F. Cannata, J.P. Dedonder, Int. J. Mod. Phys A 10, 2683 (1995);

V.G. Bagrov and B.F. Samsonov, Phys. Part. Nucl. 28, 374 (1997);

[4] D.J. Fernández, Int. J. Mod. Phys A 12, 171 (1997);

D.J. Fernández, M.L. Glasser and L.M. Nieto, Phys. Lett A 240, 15 (1998);

J.F. Cariñena, A. Ramos and D.J. Fernández, Ann. Phys. (N.Y.), 292, 42 (2001)

[5] D.J. Fernández, V. Hussin and B. Mielnik, Phys. Lett. A 244, 309 (1998)

[6] O. Rosas-Ortiz, J. Phys. A 31, L507 (1998);

O. Rosas-Ortiz, J. Phys. A 31, 10163 (1998) 
[7] I.F. Márquez, J. Negro and L.M. Nieto, J. Phys. A 31, 4115 (1998);

J.I. Díaz, J. Negro, L.M. Nieto and O. Rosas-Ortiz, J. Phys. A 32, 8447 (1999); J. Negro, L.M. Nieto and O. Rosas-Ortiz, in Foundations of Quantum Physics, R. Blanco et. al. (Eds.), p 259, Real Sociedad Española de Física (2001), Madrid, Spain

[8] B. Mielnik, L.M. Nieto and O. Rosas-Ortiz, Phys. Lett. A 269, 70 (2000)

[9] D.J. Fernández, J. Negro and L.M. Nieto, Phys. Lett. A 275, 338 (2000); J. Negro, L.M. Nieto and D.J. Fernández, Czech. J. Phys. 50, 1303 (2000); D.J. Fernández, B. Mielnik, O. Rosas-Ortiz and B.F. Samsonov, Phys. Lett. A 294, 168 (2002);

D.J. Fernández, B. Mielnik, O. Rosas-Ortiz and B.F. Samsonov, J. Phys. A 35, 4279 (2002)

[10] N. Aizawa and H-T Sato, Prog. Theor. Phys. 98, 707 (1997);

J. Jayaraman, R. de Lima Rodrigues and A.N. Vaidya, J. Phys. A 32, 6643 (1999);

J. Negro, L.M. Nieto and O. Rosas-Ortiz, J. Math. Phys. 41, 7964 (2000);

H. Aoyama, M. Sato and T. Tanaka, Phys. Lett. B 503, 423 (2001);

S. Klishevich and M. Plyushchay, Nucl. Phys. B 606, 583 (2001);

M. Sato and T. Tanaka, J. Math. Phys. 43, 3484 (2002);

B. Roy and P. Roy, Phys. Lett. A 296, 187 (2002)

[11] D.J. Fernández and E. Salinas-Hernández, J. Phys. A 36, 2537 (2003)

[12] V.G. Bagrov, I.N. Ocharov and B.F. Samsonov, J. Moscow Phys. Soc. 5, 191 (1995):

F. Cannata, G. Junker, J. Trost, Phys. Lett. A 246, 219 (1998);

A.A. Andrianov, M.V. Ioffe, F. Cannata and J.P. Dedonder, Int. J. of Mod. Phys. A 14, 2675 (1999);

B. Bagchi, S. Mallik and C. Quesne, Int. J. Mod. Phys. A 16, 2859 (2001);

B. Bagchi, S. Mallik and C. Quesne, Int. J. Mod. Phys. A 17, 51 (2002)

[13] D.J. Fernández, R. Muñoz and A. Ramos, Phys. Lett. A 308, 11 (2003)

[14] D. Baye, G. Levai and J-M Sparenberg, Nucl. Phys. A 599, 435 (1996).

[15] J.P. Malrien, P. Durand and J.P. Daudey, J. Phys. A, 18, 809 (1985)

[16] N. Hatano and D.R. Nelson, Phys. Rev. Lett. 77, 570 (1996)

[17] C.M. Bender and K. Milton, Phys Rev D 55, 3255 (1997)

[18] B.J. Hoenders, J. Phys. A 12, 2337 (1979);

F. Verheest, J. Phys. A 20, 103 (1987);

J. Larsson, Phys. Rev. Lett 66, 1466 (1991); 
A.J. Bizard, D.R. Cook and A.N. Kaufman, Phys. Rev. Lett. 70, 521 (1993);

J. Beckers, N. Debergh, J.F. Cariñena and G. Marmo, Mod. Phys. Lett. A 16, 91 (2001);

A. Ramírez and B. Mielnik, e-print: quant-ph/0211048

[19] C.M. Bender and S. Boettcher, Phys. Rev. Lett. 80, 5243 (1998)

[20] C.M. Bender, S. Boettcher and P.N. Meisinger, J. Math. Phys. 40, 2201 (1999); G. Lévai and M. Znojil, J. Phys. A 33, 7165 (2000);

R.S. Kaushal and Partnasarathi, J. Phys. A 35, 8743 (2002);

C.M. Bender, M.V. Berry and A. Mandilara, J. Phys. A 35, L467 (2002)

[21] P. Dorey, C. Dunning and R. Tateo, J. Phys A 34, 5679 (2001);

P. Dorey, C. Dunning and R. Tateo, J. Phys A 34, L391 (2001)

[22] A. Mostafazadeh, J. Math. Phys. 43, 205 (2002);

A. Mostafazadeh, J. Math. Phys. 43, 2814 (2002);

A. Mostafazadeh, J. Math. Phys. 43, 3944 (2002);

A. Mostafazadeh, Nucl. Phys. B 640, 419 (2002)

[23] A. Ahmed, Phys. Lett. A 294, 287 (2002)

[24] R. Kretschmer and L. Szymanowski, e-print: quant-ph/0105054 V1

[25] N. Gisin, J. Phys. A 14, 2259 (1981);

W. Daniel, Helv. Phys. Acta 55, 330 (1982);

B. Huttner, A. Muller, J. D. Gautier, H. Zbinden and N. Gisin, Phys. Rev. A 54, 3783 (1996)

[26] D.J. Fernández, Lett. Math. Phys. 8, 337 (1984);

B.F. Samsonov, J. Phys. A 28, 6989 (1995);

G. Junker and P. Roy, Annals Phys. 270, 155 (1998)

[27] B. Bagchi and C. Quesne, Phys. Lett. A 300, 18 (2002). 